\section{(2) OPEN ACCESS}

\title{
Non-surgical and surgical treatments for rotator cuff disease: a pragmatic randomised clinical trial with 2-year follow-up after initial rehabilitation
}

\author{
Sanna Cederqvist (D) , ${ }^{1}$ Tapio Flinkkilä, ${ }^{2}$ Markus Sormaala, ${ }^{3}$ Jari Ylinen, ${ }^{4}$ \\ Hannu Kautiainen, ${ }^{5,6}$ Tero Irmola, ${ }^{1}$ Heidi Lehtokangas, ${ }^{1,7}$ Juho Liukkonen, ${ }^{8}$ \\ Konsta Pamilo, ${ }^{9}$ Tero Ridanpää, ${ }^{9}$ Kai Sirniö, ${ }^{2}$ Juhana Leppilahti, ${ }^{2}$ Ilkka Kiviranta, ${ }^{10,11}$ \\ Juha Paloneva ${ }^{9,12}$
}

Handling editor Josef $S$ Smolen

- Additional material is published online only. To view, please visit the journal online (http://dx.doi.org/10.1136/ annrheumdis-2020-219099).

For numbered affiliations see end of article.

Correspondence to Dr Sanna Cederqvist, Department of Orthopaedics and Traumatology, Tampere University Hospital, Tampere, Finland;

sanna.cederqvist@icloud.com

Received 12 September 2020 Revised 14 November 2020 Accepted 16 November 2020 Published Online First 3 December 2020

\section{ABSTRACT}

Background Rotator cuff disease (RCD) causes prolonged shoulder pain and disability in adults. RCD is a continuum ranging from tendinopathy to full-thickness tendon tear. Recent studies have shown that subacromial decompression and non-surgical treatments provide equivalent results in RCD without a full-thickness tendon lesion. However, the importance of surgery for fullthickness tendon tears remains unclear.

Methods In a pragmatic, randomised, controlled trial, 417 patients with subacromial pain underwent 3-month initial rehabilitation and MRI arthrography (MRA) for the diagnosis of RCD. Of these, 190 shoulders remained symptomatic and were randomised to non-surgical or surgical treatments. The primary outcomes were the mean changes in the Visual Analogue Scale for pain and the Constant Murley Score for shoulder function at the 2-year follow-up.

Results At the 2-year follow-up, both non-surgical and surgical treatments for RCD reduced pain and improved shoulder function. The scores differed between groups by $4(95 \% \mathrm{Cl}-3$ to $10, \mathrm{p}=0.25)$ for pain and $3.4(95 \% \mathrm{Cl}$

-0.4 to $7.1, p=0.077)$ for function. Among patients with full-thickness ruptures, the reduction in pain $(13,95 \% \mathrm{Cl}$ 5 to $22, p=0.002$ ) and improvement in function (7.0, $95 \% \mathrm{Cl} 1.8$ to $12.2, \mathrm{p}=0.008)$ favoured surgery. Conclusions Non-surgical and surgical treatments for RCD provided equivalent improvements in pain and function. Therefore, we recommend non-surgical treatment as the primary choice for patients with RCD. However, surgery yielded superior improvement in pain and function for full-thickness rotator cuff rupture. Therefore, rotator cuff repair may be suggested after failed non-surgical treatment.

Trial registration details ClinicalTrials.gov, NCT00695981 and NCT00637013.

Check for updates

(C) Author(s) (or their employer(s)) 2021. Re-use permitted under CC BY-NC. No commercial re-use. See rights and permissions. Published by BMJ.

To cite: Cederqvist $\mathrm{S}$, Flinkkilä T, Sormaala M, et al. Ann Rheum Dis 2021;80:796-802.

\section{INTRODUCTION}

Among adults, rotator cuff disease (RCD) is the most common cause of prolonged shoulder pain and disability, ${ }^{1}$ which represent substantial healtheconomic burdens for society. ${ }^{2}$ RCD typically manifests as shoulder pain and dysfunction, ${ }^{3-5}$ and has a multifactorial aetiology, including intrinsic (eg, genetics), extrinsic (eg, trauma) and biopsychosocial factors. ${ }^{67}$ In RCD, tendon damage occurs in a continuum of acute-to-chronic changes, which

\section{Key messages}

What is already known about this subject?

- Recent studies show that subacromial decompression surgery and non-surgical treatments provide equivalent results in rotator cuff disease without full-thickness tendon lesion. The importance of surgery for fullthickness tendon tears remains unclear.

What does this study add?

- In our pragmatic study setting, we randomised patients with rotator cuff disease (RCD) who were symptomatic after 3-month initial rehabilitation, and measured change of shoulder pain and function after 2 years from randomisation.

How might this impact on clinical practice or future developments?

- Our study shows that the outcome of nonsurgical treatment is equivalent to surgical treatment in RCD without full-thickness tendon tear even after unsuccessful initial rehabilitation. However, surgery yields superior improvement in pain and function in patients with full-thickness rotator cuff tear if initial nonsurgical treatment is unsuccessful.

range from tendinopathy without frayed tendons to a full-thickness tendon tear. ${ }^{8-10}$

Non-surgical treatment is recommended for RCD initially. However, RCD is also frequently treated with surgery. ${ }^{9}$ 11-13 Subacromial decompression (SAD) surgery is a common procedure for treating a painful shoulder. ${ }^{12}$ Recent studies have shown that $\mathrm{SAD}$ and non-surgical treatments provide equivalent results in RCD without full-thickness tendon lesions. ${ }^{3-5}$ 14-17 Another common procedure is rotator cuff tendon repair. The annual rate for SAD and rotator cuff repair has been up to 130 per 100000 persons in Finland. ${ }^{9}{ }^{12}$ Whether surgical or non-surgical treatment is superior for full-thickness rotator cuff tears remains controversial. ${ }^{18-22}$

In this study, by conducting a pragmatic, randomised, controlled trial we aimed to compare surgical and non-surgical treatments for RCD with or without full-thickness tendon tears after unsuccessful initial rehabilitation. We used the Visual 


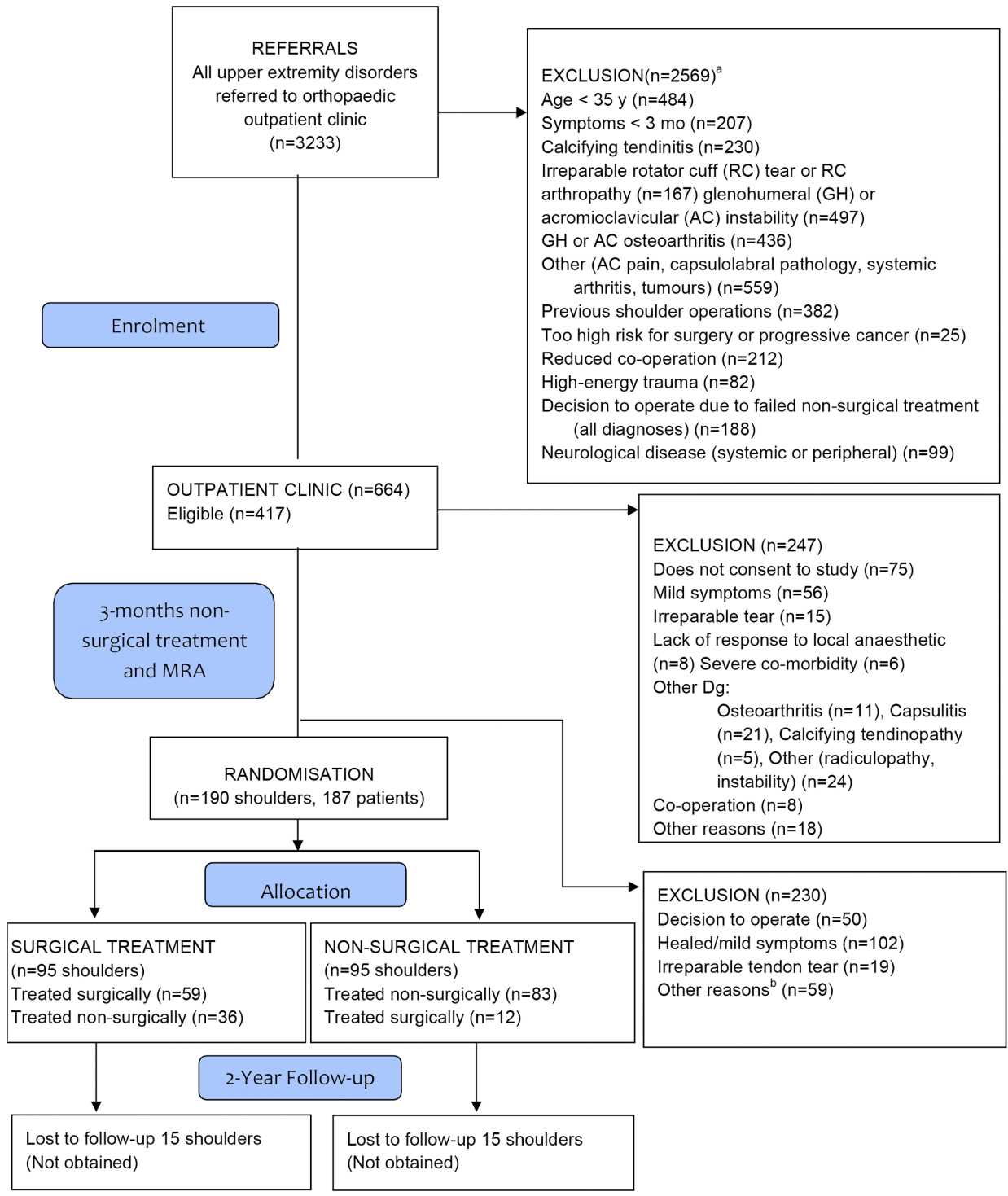

Figure 1 Trial flow chart. MRA of the shoulder. ${ }^{a}$ MRA. ${ }^{b}$ Lack of co-operation or change of diagnosis. mo, months; MRA, MRI arthrography; $y$, years.

Analogue Scale (VAS) for pain and the Constant Murley score (CMS) for function as primary outcomes.

\section{METHODS}

\section{Trial design}

This randomised, controlled, pragmatic trial was conducted in Central Finland Hospital in Jyväskylä and Oulu University Hospital.

\section{Patients}

We recruited patients with long-term (>3 months) subacromial pain who were referred from primary and occupational healthcare centres and private clinics to the two study hospitals (figure 1). Between June 2008 and December 2014, we screened 3233 referrals concerning an upper extremity disorder, including 664 who presented with symptoms attributable to RCD. The research group physicians (JP, SC, TI, JLi and HL) interviewed the patients (490 in Jyväskylä and 174 in Oulu) and performed structured examinations. Of these, 417 patients met the eligibility criteria box 1) and provided written informed consent. After inclusion, patients were advised to undergo up to 15 sessions (protocol provided in the online supplemental appendix).

After the initial 3-month non-surgical treatment, the candidates underwent MRI arthrography (MRA) and were evaluated by a specialist orthopaedic surgeon (TF, KS, KP or TR) for trial eligibility. MRA images were evaluated by clinical radiologist on duty. A full-thickness tendon tear was diagnosed if contrast medium, attributable to a full-thickness tendon tear, was detected in subacromial space in MRA. Subsequently, two study physiotherapists (one in each of the trial hospitals) randomised all suitable symptomatic patients to either non-surgical or surgical treatment (online supplemental table S1). A research assistant not involved in the study prepared a computer-generated, block randomisation list and sequentially numbered, sealed opaque envelopes for patient randomisation. We used a block size of 10 stratified according to gender and type of rotator cuff tendon lesion (RCD with or without a full-thickness tendon tear). The blocks were divided between the trial hospitals. The information regarding the treatment group was open to patients, the treating physicians and the study physiotherapists. Immediately before randomisation (baseline), the physiotherapists evaluated the primary and secondary study outcomes. 


\section{Box 1 Inclusion and exclusion criteria}

Inclusion criteria for all patients
Pain in abduction of the shoulder
Age over 35 years
Duration of symptoms at least 3 months
Written informed consent by the participating subject
Additional inclusion criteria
Subacromial impingement without full-thickness tendon lesion
Pain in two of the three isometric tests ( 0 or 30 degrees of
abduction or external rotation)
Subacromial injection of lidocaine significantly reduced pain
Full-thickness tendon rupture
Full-thickness rotator cuff rupture in one to three tendons
documented with MRI arthrography
Exclusion criteria
Previous surgery of the same shoulder
High-energy trauma before symptoms
Inflammatory arthritis
Adhesive capsulitis
Instability of the affected shoulder
Severe glenohumeral or acromioclavicular joint osteoarthritis
Cervical syndrome/radiculopathy
Progressive cancer
A too high risk for operation
Any disease, social problem or other reason reducing the ability
to co-operate and jeopardising informed consent
Irreparable rotator cuff tear on MRI arthrography

\section{Trial procedures}

Patients randomised to non-surgical treatment continued the previously initiated rehabilitation programme. Unsuccessful non-surgical treatment was defined as severe pain or poor subjective function in the shoulder during follow-up. These patients were offered a surgical intervention. In surgery, patients without full-thickness tendon tears underwent arthroscopic SAD. Patients with full-thickness tears received rotator cuff repair with single-row technique, with one or more bone anchors, via either an arthroscopic or mini-open approach. When necessary, patients underwent acromioplasty, acromioclavicular joint resection or tenotomy of the long head of the biceps. All patients followed a structured postoperative rehabilitation protocol (see online supplemental appendix).

\section{Outcomes}

The primary outcome evaluated 2 years after randomisation was the change in the intensity of pain, during the previous week, on VAS and the change in CMS for rating shoulder function. Outcome measures were recorded at baseline and 3, 6, 12 and 24 months after randomisation. VAS (0 to $100,100=$ worst possible pain) was calculated as the mean pain at rest, during arm activity and at night. The study physiotherapists measured shoulder function with CMS (scale: 1 to $100,100=$ best). ${ }^{23}$ The pain-free (VAS $<30$ ) shoulder range of motion was measured by a goniometer. ${ }^{24}$

With the arm abducted 90 degrees, maximal isometric shoulder abduction strength was measured with a strain-gauge dynamometer (DS Europe, Milan, Italy) at Jyväskylä or a spring scale at Oulu. The sensor was placed at the level of the styloid process of the radius. We used a modified method of measuring the strength by calculating the mean of three efforts instead of the best out of three as described by Constant. ${ }^{23}$ Abduction strength was rated zero when the patient could not achieve the measuring position at 90 -degree abduction.

The secondary outcome was the health-related quality of life as measured with the RAND 36-Item Health Survey. ${ }^{25}$ We also recorded serious adverse events and re-operations.

\section{Statistical analysis}

The appropriate sample size was estimated with a simulationbased model. Calculations were based on a $30 \%$ difference in pain between treatment groups. When significant, a 30\% difference was also likely to be clinically relevant. ${ }^{4}$ We determined that approximately 200 patients (100 per research arm) were required for a two-sided significance level of 0.05 ( $85 \%$ power).

All primary analyses were performed based on the intentionto-treat (ITT) principle. Data are expressed as the mean and SD, the median and IQR or counts and percentages, as appropriate. The non-surgery and surgery groups were compared using the t-test for continuous variables and Pearson's $\chi^{2}$ test for categorical variables in baseline values. Repeated measures of the changes in primary (ITT and per protocol, PP) and secondary outcomes (ITT) were compared between the non-surgery and surgery groups with mixed-effects models and an unstructured covariance structure (ie, the Kenward-Roger method for calculating the df). ${ }^{26}$ Fixed effects included group, time and group $x$ time interactions. We used baseline values as covariates when appropriate. The repeated measurements were taken at different time points, including baseline, 3, 6, 12 and 24 months. Mixed models allowed analysis of unbalanced datasets without imputation; therefore, we analysed all available data with the full analysis set. Normal distributions were evaluated graphically and with the Shapiro-Wilk W test. All analyses were performed in Stata 16.0 (StataCorp LP; College Station, Texas, USA).

Two pre-specified subgroup analyses were performed for subgroups of RCD, with or without full-thickness tendon tears.

\section{RESULTS}

\section{Recruitment}

After 3 months of pragmatic non-surgical treatment, 247 patients were excluded due to reasons shown in trial flow chart (figure 1) and 187 patients (190 shoulders) randomised (table 1).

Group allocation (full-thickness or non-full-thickness tendon lesion) was based on written statement made by clinical radiologist. Of these, 95 shoulders were randomised to receive surgery (50 shoulders with full-thickness ruptures, of which 44 solely in the supraspinatus tendon) and 95 to non-surgical treatments (48 with full-thickness ruptures, of which 44 were solely in the supraspinatus tendon). In the non-surgery group, 12 (13\%) shoulders experienced severe pain and surgery was performed during the 2-year follow-up. In the surgery group, 36 (38\%) shoulders experienced pain relief before surgery and did not undergo surgery. Shoulders treated per protocol were $75 \%$ (figure 2). Online supplemental table S1 shows the frequency of missing data.

\section{Primary outcomes}

At the 2-year follow-up, the mean VAS score decreased by 31 (95\% CI 26 to 35$)$ in the non-surgery group and by 34 (95\% CI 30 to 39) in the surgery group. The difference between groups was not significant (mean difference: $4,95 \% \mathrm{CI}-3$ to 10 ; $\mathrm{p}=0.25)$. The mean Constant score (CS) improved by $17.0(95 \%$ CI 14.4 to 19.7$)$ in the non-surgery group and by $20.4(95 \%$ CI 17.8 to 23.1 ) in the surgery group. The difference between 
Table 1 Baseline characteristics of patients with rotator cuff disease

\begin{tabular}{|c|c|c|}
\hline & $\begin{array}{l}\text { Non-surgical group } \\
(n=95)^{*}\end{array}$ & $\begin{array}{l}\text { Surgical group } \\
(n=95)^{*}\end{array}$ \\
\hline Patients & 93 & 94 \\
\hline Men, n (\%) & $50(53)$ & $52(55)$ \\
\hline Full-thickness ruptures, n (\%) & $48(51)$ & $50(53)$ \\
\hline Right shoulder, n (\%) & $62(65)$ & $58(61)$ \\
\hline Age, mean (SD) & $56(8)$ & $56(8)$ \\
\hline Duration of pain, months, median (IQR) & 12 (8 to 21$)$ & 12 (8 to 36) \\
\hline Traumatic onset & $16(17)$ & $17(18)$ \\
\hline \multicolumn{3}{|l|}{ Pain, VAS, mean (SD) } \\
\hline Rest & $37(26)$ & $36(25)$ \\
\hline Arm activity & $60(23)$ & $55(26)$ \\
\hline Night & $51(29)$ & $50(28)$ \\
\hline Constant score, mean (SD) & $55(16)$ & $57(17)$ \\
\hline \multicolumn{3}{|l|}{ Had performed exercises } \\
\hline PT-guided exercises n (\%) & $60(63)$ & $53(56)$ \\
\hline Home exercises, n (\%) & $52(55)$ & $44(46)$ \\
\hline Received corticosteroid injections, n (\%) & $65(68)$ & $67(71)$ \\
\hline
\end{tabular}

*Shoulders.

PT, physiotherapist; VAS, Visual Analogue Scale.

groups was not significant (mean difference: $3.4,95 \% \mathrm{CI}-0.4$ to $7.1 ; \mathrm{p}=0.077$; figure 3 and online supplemental table S2).

Among patients without full-thickness rotator cuff ruptures at the 2-year follow-up, the mean VAS decreased by 38 (95\% CI 31 to 45$)$ in the non-surgery group and by 31 (95\% CI 24 to 38 ) in the surgery group. The difference between groups was not significant (mean difference: $7,95 \% \mathrm{CI}-3$ to $17 ; \mathrm{p}=0.19$; figure 3 and online supplemental table S2). The mean CS improved by 21.6 (95\% CI 17.8 to 25.3 ) in the non-surgery group and by 20.9 (95\% CI 17.1 to 24.7 ) in the surgery group. The difference between groups was not significant (mean difference: 0.7 , $95 \% \mathrm{CI}-4.6$ to $6.1 ; \mathrm{p}=0.79$ ).

Among patients with full-thickness ruptures at the 2-year follow-up, the mean VAS score decreased by 24 (95\% CI 18 to

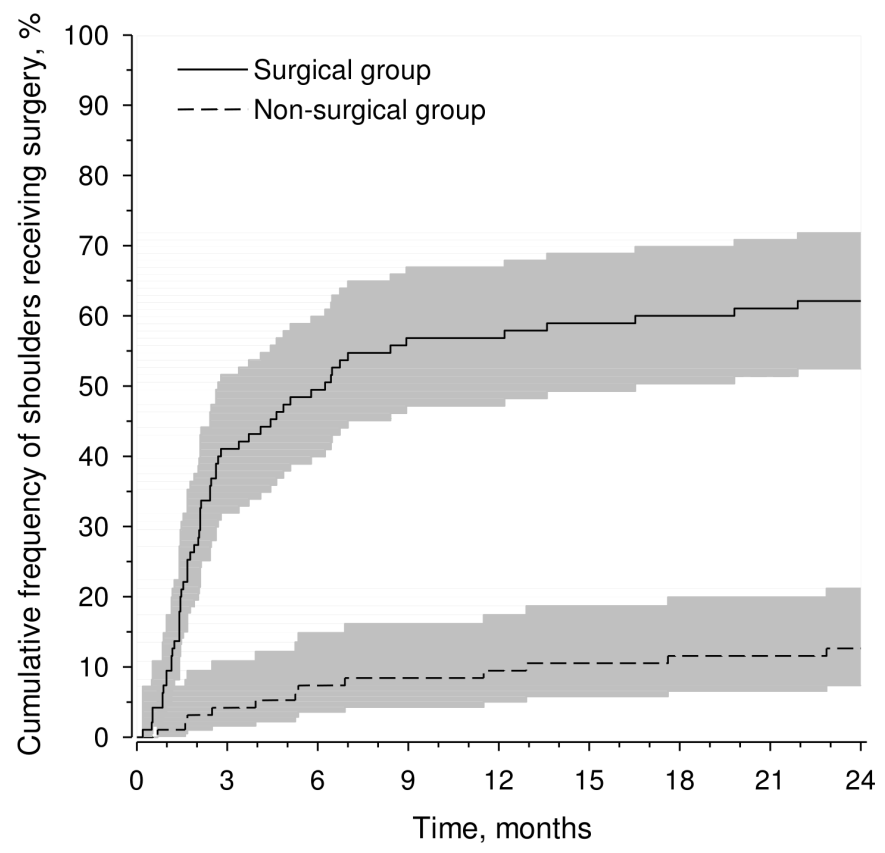

Figure 2 Cumulative frequency of shoulders receiving surgery in surgical and non-surgical randomisation groups with $95 \% \mathrm{Cls}$.
$30)$ in the non-surgery group and by 37 (95\% CI 31 to 43) in the surgery group. The difference between groups was significant (mean difference: $13,95 \%$ CI 5 to $22 ; \mathrm{p}=0.002$ ). The mean CS improved by 13.0 (95\% CI 9.4 to 16.7 ) in the non-surgery group and by 20.0 (95\% CI 16.4 to 23.7 ) in the surgery group. The difference between groups was significant (mean difference: $7.0,95 \%$ CI 1.8 to $12.2 ; \mathrm{p}=0.008$ ). The PP results are shown in Supplements (online supplemental figure S1).

\section{Health-related quality of life}

At the 2-year follow-up, the changes in mean RAND-36 scores for physical function, general health, vitality, role physical, role emotional, social functioning and bodily pain were not significantly different between the non-surgery and surgery groups. Among patients without full-thickness rotator cuff ruptures, the changes of quality of life were similar in the non-surgery and surgery groups. Among patients with full-thickness rotator cuff ruptures, the bodily pain score improved 13 points (95\% CI 3 to $23 ; p=0.011)$ more in the surgery group than in the non-surgery group (table 2 ).

\section{Adherence to non-surgical treatment modalities}

During the 2-year follow-up, 38\% of all patients underwent physiotherapy, $46 \%$ performed home-based exercise and $8 \%$ received corticosteroid injections. The implementation of home-based exercise was similar between the treatment groups. However, the surgery group had a higher frequency of physiotherapy visits than the non-surgery group $(p<0.001)$, and the non-surgery group received more corticosteroid injections $(\mathrm{p}=0.015$; online supplemental table S3).

\section{Adverse events}

No patients required re-operation, and no serious adverse events were noted.

\section{DISCUSSION}

We found equivalent improvements in pain and function at the 2-year follow-up in both treatment groups, and changes in quality of life were not significantly different between the two treatment groups. Similar results were found in the subgroup of patients without full-thickness ruptures. This result is consistent with previous trials of patients with more acute, undefined, initial non-surgical treatment. ${ }^{3-5} 14192127$

Among patients with full-thickness rotator cuff ruptures at the 2-year follow-up, pain relief was better with surgery than nonsurgical treatment. In this subgroup, the two treatments produced significantly different changes in mean pain, pain at rest and pain during the night. In contrast, both treatments had similar effects on pain related to arm activity. The bodily pain dimension in the RAND-36 quality of life questionnaire improved more with surgery than non-surgical treatments. These findings contrast with recent findings from Kukkonen et al, but support earlier findings from Moosmayer et al. ${ }^{18} 20$ At the 2-year follow-up, shoulder function improved with both treatments, but the CS improved 7 points more with surgery than non-surgical treatments. The minimum clinically important difference between groups has not been determined unequivocally. ${ }^{3} 2829$

Due to the pragmatic approach of the study, exercise compliance was relatively low (online supplemental table S3). Patients had experienced shoulder pain for relatively long periods of time, and many had received physiotherapy before recruitment(table 1). Thus, patients were familiar with the exercise methods, which may explain the relatively low attendance at 

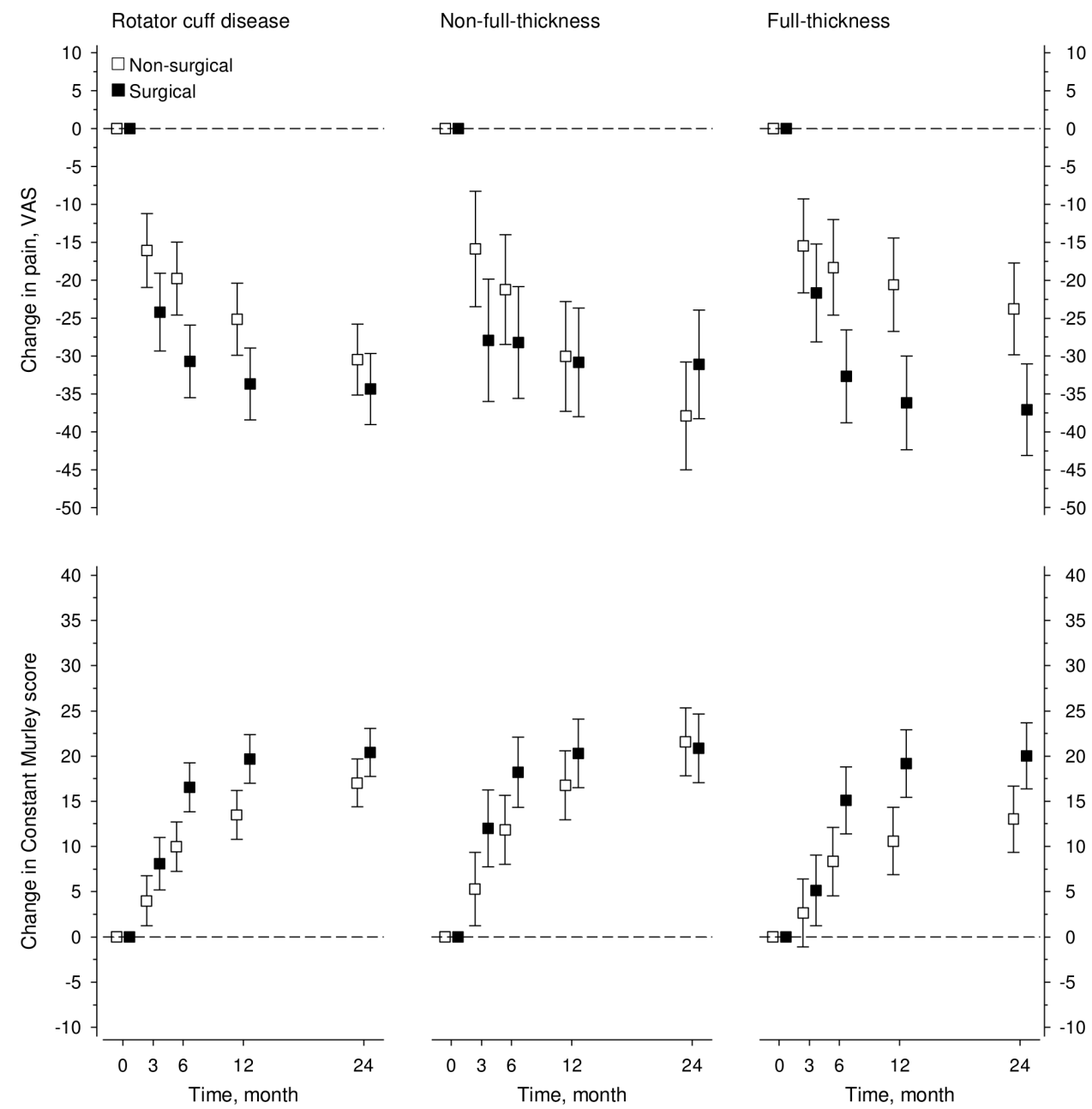

Figure 3 Graphs showing the change in pain in the visual analogue scale (VAS, mm) and the Constant score between baseline and the 2-year follow-up in all patients with rotator cuff disease and without and with full-thickness rotator cuff rupture.

the recommended physiotherapy. In our study design, nonsurgical treatment was comparable to ordinary practice. Patients in the surgery group attended physiotherapy significantly more frequently than patients in the non-surgery group. In the nonsurgery group, the use of corticosteroid injections was higher compared with the surgery group. Our patients presented with broad RCD aetiology because we initially merely excluded patients with high-energy traumas and irreparable tendon tears. Moreover, we analysed RCD subgroups of patients with and without full-thickness tears.

None of the five previously published trials reported difference in surgical and non-surgical treatments for patients with RCD without full-thickness rotator cuff tears (impingement). ${ }^{3-5}$ In a 2.5 -year follow-up study, Brox et al found that surgery outcomes were not significantly different from outcomes after 3 to 6 months of intensive, supervised exercise. ${ }^{3}$ Similarly, in a 4 to 8 year follow-up study, Haahr et al found that surgery outcomes were not different from outcomes after intensive supervised physiotherapy. ${ }^{4}$ Ketola et al found similar outcomes with supervised exercise treatment versus SAD followed by supervised exercises. ${ }^{5}$ Two recent controlled trials and a meta-analysis of subacromial decompression efficacy in patients with RCD found no difference in VAS pain scores after subacromial decompression, placebo arthroscopy or exercise therapy. ${ }^{14-16}$

Five randomised controlled trials compared surgical and non-surgical treatments for full-thickness supraspinatus tendon ruptures. ${ }^{18-2022}$ Moosmayer et al found between-group differences that slightly favoured surgery based on the VAS for pain, the CS and the American Shoulder and Elbow Surgeons Shoulder Score during a 5-year follow-up. ${ }^{19}$ Another study by Kukkonen et al found no difference between surgical and nonsurgical treatments for non-traumatic supraspinatus tears. ${ }^{27}$ Lambers Heerspink et al detected significant improvements in degenerative rotator cuff tears at a 1 -year follow-up that favoured surgery over conservative treatment based on mean scores from the Dutch Simple Shoulder Test and a VAS. However, the CS values were similar between treatment groups. ${ }^{22}$ Odak et al assessed the efficacy of SAD with or without mini-open cuffrepair, yielding no difference between the groups at a 1-year follow-up.$^{30}$ A study of Ranebo et al compared the non-surgical and surgical treatment of small, acute traumatic supraspinatus tears reporting uniform findings between the groups. ${ }^{31}$ Among these randomised, controlled trials, only two included traumatic tears. ${ }^{1931}$ In our study, $17 \%$ of patients attributed their shoulder problems to low-energy traumas. Generally, traumatic rotator cuff tears are considered an indication for tendon repair, but this lacks solid scientific evidence. ${ }^{31}$ In contrast, non-traumatic rotator cuff tears are often treated conservatively. Tendon degeneration has been demonstrated in a majority of rotator cuff tendon tears. ${ }^{10} 32$ The different aetiologies of rotator cuff tears may explain the contradictory findings among previous studies.

No previous studies investigated the effectiveness of surgery after adequately performed, but unsuccessful, non-surgical treatment of RCD including both non-full-thickness and full-thickness 
Table 2 36-item short form health survey questionnaire (RAND-36) for health-related quality of life

\begin{tabular}{|c|c|c|c|c|c|c|}
\hline & \multicolumn{2}{|l|}{ Baseline } & \multicolumn{2}{|c|}{ Change from baseline to months 24} & \multicolumn{2}{|c|}{$P$ values between groups } \\
\hline & $\begin{array}{l}\text { Non-surgical } \\
\text { Mean (SD) }\end{array}$ & $\begin{array}{l}\text { Surgical } \\
\text { Mean (SD) }\end{array}$ & $\begin{array}{l}\text { Non-surgical } \\
\text { Mean }(95 \% \mathrm{Cl})\end{array}$ & $\begin{array}{l}\text { Surgical } \\
\text { Mean }(95 \% \mathrm{Cl})\end{array}$ & Crude & Adjusted* \\
\hline \multicolumn{7}{|c|}{ Rotator cuff disease (all) $n=190$} \\
\hline Physical function & $72(19)$ & $75(16)$ & 5 (2 to 9$)$ & 5 (2 to 8$)$ & 0.78 & 0.65 \\
\hline General health & $59(17)$ & $62(19)$ & $3(-1$ to 6$)$ & $1(-2$ to 4$)$ & 0.48 & 0.42 \\
\hline Vitality & $62(21)$ & $63(21)$ & 6 (2 to 9$)$ & $7(3$ to 10$)$ & 0.73 & 0.89 \\
\hline Mental health & $74(18)$ & $76(18)$ & 4 (1 to 7$)$ & 5 (2 to 7$)$ & 0.37 & 0.61 \\
\hline Role physical & $38(40)$ & $44(39)$ & 22 (13 to 32 ) & 23 (14 to 32$)$ & 0.98 & 0.99 \\
\hline Emotional role & $73(39)$ & $71(41)$ & $2(-7$ to 10$)$ & $9(2$ to 17$)$ & 0.18 & 0.16 \\
\hline Social function & $74(22)$ & $80(23)$ & 8 (3 to 12$)$ & 5 (1 to 9$)$ & 0.41 & 0.17 \\
\hline Bodily pain & $44(19)$ & $43(19)$ & 15 (10 to 21$)$ & 21 (16 to 26$)$ & 0.11 & 0.15 \\
\hline \multicolumn{7}{|c|}{ Non-full-thickness rupture $n=92$} \\
\hline Physical function & $70(23)$ & $74(18)$ & 10 (4 to 16$)$ & $3(-3$ to 8$)$ & 0.065 & 0.063 \\
\hline General health & $57(19)$ & $59(19)$ & $4(-1$ to 9$)$ & $1(-3$ to 6$)$ & 0.47 & 0.32 \\
\hline Vitality & $59(22)$ & $58(21)$ & 8 (2 to 14$)$ & 10 (4 to 15$)$ & 0.65 & 0.92 \\
\hline Mental health & $73(18)$ & $75(18)$ & $4(-1$ to 9$)$ & $6(2$ to 10$)$ & 0.61 & 0.58 \\
\hline Role physical & $39(43)$ & $43(38)$ & 30 (15 to 45$)$ & 21 (9 to 34$)$ & 0.39 & 0.43 \\
\hline Emotional role & $70(43)$ & $65(43)$ & $12(-1$ to 25$)$ & 17 (6 to 28$)$ & 0.54 & 0.51 \\
\hline Social function & $76(23)$ & $80(21)$ & $8(0$ to 15$)$ & 6 (0 to 12$)$ & 0.75 & 0.42 \\
\hline Bodily pain & $41(20)$ & $41(20)$ & 22 (13 to 30$)$ & 20 (12 to 27$)$ & 0.72 & 0.46 \\
\hline \multicolumn{7}{|c|}{ Full-thickness rupture $n=98$} \\
\hline Physical function & $74(16)$ & $76(14)$ & $2(-2$ to 6$)$ & 7 (3 to 11$)$ & 0.078 & 0.096 \\
\hline General health & $60(16)$ & $64(19)$ & 2 (-2 to 6$)$ & $1(-3$ to 5$)$ & 0.77 & 0.91 \\
\hline Vitality & $63(20)$ & $67(20)$ & $4(0$ to 8$)$ & 4 (0 to 8 ) & 0.82 & 0.73 \\
\hline Mental health & $75(18)$ & $78(18)$ & $4(0$ to 8$)$ & 4 (0 to 8 ) & 0.98 & 0.82 \\
\hline Role physical & $38(38)$ & $44(39)$ & 17 (3 to 30$)$ & 24 (11 to 37 ) & 0.43 & 0.44 \\
\hline Emotional role & $75(34)$ & $77(38)$ & $-6(-17$ to 5$)$ & $2(-9$ to 12$)$ & 0.30 & 0.32 \\
\hline Social function & $73(22)$ & $81(25)$ & 8 (2 to 14$)$ & $4(-2$ to 10$)$ & 0.39 & 0.26 \\
\hline Bodily pain & $47(17)$ & $44(18)$ & 10 (3 to 17$)$ & 23 (16 to 30$)$ & 0.011 & 0.006 \\
\hline
\end{tabular}

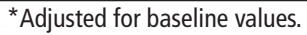

tendon lesions. In our trial, all potential participants underwent a structured, 3-month rehabilitation before randomisation; thus, only symptomatic patients were randomised. In addition, the flow of patients referred to specialised care was trackable, and our study approach was pragmatic. We submitted two clinical trial registries (one subgroup for subacromial impingement syndrome stage II and another for full-thickness tendon ruptures) because at the time of registration (2008) it was not generally accepted that rotator cuff disease is an actual continuum that ranges from subacromial impingement syndrome to a full-thickness rotator cuff tendon rupture.

Due to the pragmatic approach, it was reasoned to analyse these two types of tendon lesions together. We minimised the potential influence of technical differences between surgeons for recruitment and surgery; five physicians recruited the patients and five surgeons performed the surgeries. Therefore, our findings can be readily applied to clinical practice.

Our study lacked a placebo surgery group and the study physiotherapists were not blinded. A potential source of bias is that we included three patients twice. The shoulders are not independent when reporting pain and disability. Only $63 \%$ of patients who underwent clinical examination were eligible for this study, and $55 \%$ of the shoulders preliminarily meeting the inclusion criteria were excluded from randomisation, mostly due to relief of symptoms. Thus, our primary results are only applicable to patients who do not recover after initial non-surgical treatment. Moreover, $26 \%$ of patients were not treated PP. The results of the PP analysis supported the results of the ITT analysis. Surgery yielded superior results in the change of shoulder pain and the function compared with non-surgical management in PP analyses.

\section{CONCLUSIONS}

Our results demonstrate that surgery does not provide superior results compared with non-surgical treatment for the majority of patients with RCD. Among patients with symptomatic RCD without a perforating tear, surgery did not provide benefit over non-surgical treatment, even when the initial non-surgical treatment did not provide sufficient pain relief. However, when the RCD included a perforating tear and symptoms continued after initial non-surgical treatment, rotator cuff repair surgery resulted in superior outcomes compared with non-surgical treatment.

\section{Author affiliations}

${ }^{1}$ Department of Orthopaedics and Traumatology, Tampere University Hospital, Tampere, Finland

${ }^{2}$ Department of Surgery, Division of Orthopaedic and Trauma Surgery, Medical Research Center, Oulu University Hospital, Oulu, Finland

${ }^{3}$ Department of Radiology, Helsinki University Hospital, Helsinki, Finland

${ }^{4}$ Department of Physical Medicine and Rehabilitation, Central Finland Central Hospital, Jyväskylä, Finland

${ }^{5}$ Research Center, Folkhälsan, Helsinki, Finland

${ }^{6}$ Primary Health Care Unit, Kuopio University Hospital, Kuopio, Finland

${ }^{7}$ Faculty of Medicine and Health Technology, Tampere University, Tampere, Finland

${ }^{8}$ Department of Emergency Medicine, Central Finland Central Hospital, Jyväskylä, Finland

${ }^{9}$ Department of Surgery, Central Finland Central Hospital, Jyväskylä, Finland 
${ }^{10}$ Department of Orthopaedics and Traumatology, Helsinki University Hospital, Helsinki, Finland

${ }^{11}$ Department of Orthopaedics and Traumatology, University of Helsinki, Helsinki, Finland

${ }^{12}$ Institute of Clinical Medicine, University of Eastern Finland, Kuopio, Finland

Correction notice This article has been corrected since it published Online First. The first supplemental file has been replaced.

Acknowledgements We wish to thank all of the participants in the trial and the organisations and personnel. The research group thanks our study physiotherapists Saara-Maija Hinkkanen and Nina Sevander-Kreus for their support.

Contributors Concept and design: JP, IK, HK and JY. Acquisition analysis or data interpretation: SC, TF, MS, JY, HK, IK, JP, TI, HL, JLe, JLi, KP, TR and KS. SC and JP had full access to all the data in the study and take responsibility for the integrity of the data and the accuracy of the data analysis. Drafting the manuscript: SC, TF, MS, JY, HK, IK and JP. Critical revision of the manuscript for important intellectual content: SC, TF, MS, JY, HK, IK, JP, TI, HL, JLe, JLi, KP, TR and KS. Statistical analysis: HK, SC and JP. Funding procurement: JP, IK and JLe. Administrative, technical or material support: Study physiotherapists: Saara-Maija Hinkkanen and Nina Sevander-Kreus. Supervision: JP, IK and HK.

Funding This work was supported by grants from the Academy of Finland (grant 12321/13.9.2007) 265646/17.4.2013) and National Competitive Research Funding of the University of Eastern Finland. None of the writers have any conflicts of interest relevant to this article.

Competing interests None declared.

Patient consent for publication Not required.

Ethics approval The Ethics Committee of the Central Finland Health Care District approved the trial on 23 May 2007.

Provenance and peer review Not commissioned; externally peer reviewed.

Data availability statement Data sharing not applicable as no data sets generated and/or analysed for this study. Study data cannot be shared publicly because of confidentiality requirements under Finnish law. De-identified patient data is available from the study group and from Central Finland Hospital (contact via www.ksshp.fi, juha.paloneva@ksshp.fi) for researchers who meet the criteria for access to confidential data.

Supplemental material This content has been supplied by the author(s). It has not been vetted by BMJ Publishing Group Limited (BMJ) and may not have been peer-reviewed. Any opinions or recommendations discussed are solely those of the author(s) and are not endorsed by BMJ. BMJ disclaims all liability and responsibility arising from any reliance placed on the content. Where the content includes any translated material, BMJ does not warrant the accuracy and reliability of the translations (including but not limited to local regulations, clinical guidelines, terminology, drug names and drug dosages), and is not responsible for any error and/or omissions arising from translation and adaptation or otherwise.

Open access This is an open access article distributed in accordance with the Creative Commons Attribution Non Commercial (CC BY-NC 4.0) license, which permits others to distribute, remix, adapt, build upon this work non-commercially, and license their derivative works on different terms, provided the original work is properly cited, appropriate credit is given, any changes made indicated, and the use is non-commercial. See: http://creativecommons.org/licenses/by-nc/4.0/

ORCID iD

Sanna Cederqvist http://orcid.org/0000-0002-5060-4641

\section{REFERENCES}

1 Mitchell C, Adebajo A, Hay E, et al. Shoulder pain: diagnosis and management in primary care. BMJ 2005;331:1124-8

2 Oh LS, Wolf BR, Hall MP, et al. Indications for rotator cuff repair: a systematic review. Clin Orthop Relat Res 2007:455:52-63.

3 Brox Jl, Gjengedal E, Uppheim G, et al. Arthroscopic surgery versus supervised exercises in patients with rotator cuff disease (stage II impingement syndrome): a prospective, randomized, controlled study in 125 patients with a 2 1/2-year follow-up. J Shoulder Elbow Surg 1999:8:102-11.

4 Haahr JP, Østergaard S, Dalsgaard J, et al. Exercises versus arthroscopic decompression in patients with subacromial impingement: a randomised, controlled study in 90 cases with a one year follow up. Ann Rheum Dis 2005;64:760-4.
5 Ketola S, Lehtinen J, Arnala I, et al. Does arthroscopic acromioplasty provide any additional value in the treatment of shoulder impingement syndrome?: a two-year randomised controlled trial. J Bone Joint Surg Br 2009;91:1326-34.

6 Seitz AL, McClure PW, Finucane S, et al. Mechanisms of rotator cuff tendinopathy: intrinsic, extrinsic, or both? Clin Biomech 2011;26:1-12.

7 Brox JI. Shoulder pain. Best Pract Res Clin Rheumatol 2003;17:33-56.

8 Neer CS. Impingement lesions. Clin Orthop Relat Res 1983:70-7

9 Paloneva J, Lepola V, Äärimaa V , et al. Increasing incidence of rotator cuff repairs--A nationwide registry study in Finland. BMC Musculoskelet Disord : 2015;16:189.

10 Hashimoto T, Nobuhara K, Hamada T. Pathologic evidence of degeneration as a primary cause of rotator cuff tear. Clin Orthop Relat Res 2003;415:111-20.

11 Ryösä A, Laimi K, Äärimaa V, et al. Surgery or conservative treatment for rotator cuff tear: a meta-analysis. Disabil Rehabil 2017;39:1357-63.

12 Paloneva J, Lepola V, Karppinen J, et al. Declining incidence of acromioplasty in Finland. Acta Orthop 2015;86:220-4

13 Carr A, Cooper C, Campbell MK, et al. Effectiveness of open and arthroscopic rotator cuff repair (UKUFF): a randomised controlled trial. Bone Joint J 2017;99-B:107-15.

14 Paavola M, Malmivaara A, Taimela S, et al. Subacromial decompression versus diagnostic arthroscopy for shoulder impingement: randomised, placebo surgery controlled clinical trial. BMJ 2018;362:k2860.

15 Beard DJ, Rees JL, Cook JA, et al. Arthroscopic subacromial decompression for subacromial shoulder pain (CSAW): a multicentre, pragmatic, parallel group, placebocontrolled, three-group, randomised surgical trial. Lancet 2018;391:329-38.

16 Vandvik PO, Lähdeoja T, Ardern C, et al. Subacromial decompression surgery for adults with shoulder pain: a clinical practice guideline. BMJ 2019;364:1294.

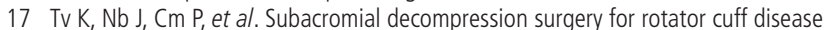
(review). Cochrane Database Syst Rev 2019;1.

18 Moosmayer S, Lund G, Seljom U, et al. Comparison between surgery and physiotherapy in the treatment of small and medium-sized tears of the rotator cuff: a randomised controlled study of 103 patients with one-year follow-up. J Bone Joint Surg Br 2010;92:83-91.

19 Moosmayer S, Lund G, Seljom US, et al. Tendon repair compared with physiotherapy in the treatment of rotator cuff tears: a randomized controlled study in 103 cases with a five-year follow-up. J Bone Joint Surg Am 2014;96:1504-14.

20 Kukkonen J, Joukainen A, Lehtinen J, et al. Treatment of nontraumatic rotator cuff tears. J Bone Joint Surg 2015;97:1729-37.

21 Kukkonen J, Joukainen A, Lehtinen J, et al. Treatment of non-traumatic rotato cuff tears: a randomised controlled trial with one-year clinical results. Bone Joint $J$ 2014;96-B:75-81.

22 Lambers Heerspink FO, van Raay JJAM, Koorevaar RCT, et al. Comparing surgical repair with conservative treatment for degenerative rotator cuff tears: a randomized controlled trial. J Shoulder Elbow Surg 2015;24:1274-81.

23 Constant $\mathrm{CR}$, Gerber $\mathrm{C}$, Emery RJH, et al. A review of the constant score: modifications and guidelines for its use. J Shoulder Elbow Surg 2008;17:355-61.

24 Zelman DC, Dukes E, Brandenburg N, et al. Identification of cut-points for mild, moderate and severe pain due to diabetic peripheral neuropathy. Pain 2005;115:29-36

25 Aalto AM, Aro AR, Teperi J. Rand-36 terveyteen liittyvän elämänlaadun mittarina: Mittarin luotettavuus JA suomalaiset väestöarvot. Research reports / National Research and Development Centre for Welfare and Health. 1999 http//urn. fiURN:NBN:fi-fe201211089642

26 Kenward MG, Roger JH. Small sample inference for fixed effects from restricted maximum likelihood. Biometrics 1997;53:983.

27 Kukkonen J, Joukainen A, Lehtinen J, et al. Treatment of nontraumatic rotator cuff tears: a randomized controlled trial with two years of clinical and imaging follow-up. $J$ Bone Joint Surg Am 2015;97:1729-37.

28 Holmgren T, Öberg B, Adolfsson L, et al. Minimal important changes in the Constant-Murley score in patients with subacromial pain. J Shoulder Elbow Surg 2014:23:1083-90.

29 Kukkonen J, Kauko T, Vahlberg T, et al. Investigating minimal clinically important difference for constant score in patients undergoing rotator cuff surgery. J Shoulder Elbow Surg 2013;22:1650-5.

30 Odak S, Powell E, Temperley D, et al. A randomized controlled trial to assess the efficacy of arthroscopic subacromial decompression with and without rotator cuff repair using a Mini-open technique. Shoulder Elbow 2012:4:112-6.

31 Ranebo MC, Björnsson Hallgren HC, Holmgren T, et al. Surgery and physiotherapy were both successful in the treatment of small, acute, traumatic rotator cuff tears: a prospective randomized trial. J Shoulder Elbow Surg 2020;29:459-70.

32 Kannus P. Etiology and pathophysiology of chronic tendon disorders in sports. Scand J Med Sci Sports 1997;7:78-85. 